\title{
The octonionic eigenvalue problem
}

\author{
Stefano De Leo ${ }^{1}$ and Gisele Ducati ${ }^{2}$ \\ ${ }^{1}$ Department of Applied Mathematics, Universidade Estadual de Campinas \\ ${ }^{2}$ Center of Mathematics, Computation and Cognition, Universidade Federal do ABC \\ E-mail: deleo@ime.unicamp.br and ducati@ufabc.edu.br \\ Journal of Physics A 45, 315203-17 (2012)
}

\begin{abstract}
By using a real matrix translation, we propose a coupled eigenvalue problem for octonionic operators. In view of possible applications in quantum mechanics, we also discuss the hermiticity of such operators. Previous difficulties in formulating a consistent octonionic Hilbert space are solved by using the new coupled eigenvalue problem and introducing an appropriate scalar product for the probability amplitudes.
\end{abstract}

PACS numbers: 02.30.Tb, 03.65.Fd 


\section{Introduction}

Developments in quaternionic matrix theory produced interesting and important results in approaching and solving quaternionic eigenvalue problems [1, 2] and linear differential equations with quaternionic coefficients [3, 4]. This renewed the interest in studying quaternionic formulations of quantum mechanics [5]. Previous discussions on quaternionic diffusion and tunnelling phenomena [6, 7, 8, 9, 10, 11] and recent analysis of confined states [12 are now based on a more solid mathematical understanding of the quaternionic structures involved in such physical problems. Consequently, many of the previous hidden aspects of the theory have been clarified and more convincing proposals of quaternionic deviations from complex theory can be now formulated. If quaternions, due to their non-commutativity, represent a challenge for mathematicians and physicists, the use of octonions to formulate quantum theories, due to their non-commutativity and non-associativity, seems a very hard challenge. It soon becomes clear that describing the physical world in terms of octonionic mathematical structures involves many conceptual (algebraic and analytic) difficulties that the lack of associativity inevitably conjures up [13, 14. In this spirit, the paper was intended as an attempt to motivate and stimulate the study of octonionic mathematical problems in view of possible applications in physical theory. The main difficulty in carrying out octoninic formulations of quantum mechanics is related to the appropriate definition of octonionic Hilbert spaces and scalar products [5, 15, 16]. In the next section, we shall come back to this point and present a detailed discussion on octonionic operators and complex geometry. We shall see that the use of basic concepts in quantum mechanics, such as the correct choice of the number system to appropriately define amplitudes of probabilities, suggests the introduction of coupled octonionic eigenvalue equations where two real parameters play the role that complex eigenvalues do in complex and quaternionic quantum mechanics.

Before to proceed with the main topic of our paper, i.e. the octonionic eigenvalue problem in quantum mechanics, let us set up notation and terminology, and study a simple eigenvalue problem by using complex, quaternionic and octonionic algebras. This shall elucidate in a practical way some of the difficulties due to the lost of commutativity and associativity.

Let $(\mathbb{O}$ be the octonionic division algebra. A generic element in this algebra will be represented by

$$
o=r_{0}+\sum_{m=1}^{7} r_{m} e_{m}, \quad r_{0,1, \cdots, 7} \in \mathbb{R},
$$

where $e_{m}$ are the octonionic immaginary units obeying the following non-commutative and non-associative algebraic rules

$$
e_{m} e_{n}=-\delta_{m n}+\epsilon_{m n p} e_{p}
$$

with $m, n, p=1,2, \cdots, 7$ and $\epsilon_{m n p}$ which is a totally antisymmetric tensor equal to the unit for the seven (quaternionic) combinations

123, 145, 176, 246, 257, 347 and 365 . 
The conjugate, the norm and the inverse of an octonion are respectively defined by

$$
\begin{aligned}
& o^{\dagger}=r_{0}-\sum_{m=1}^{7} r_{m} e_{m}, \\
& N(o)=\sqrt{o^{\dagger} O}=\sqrt{o o^{\dagger}}=\sqrt{r_{0}^{2}+\cdots+r_{7}^{2}}, \\
& o^{-1}=o^{\dagger} / N(o) \quad(0 \neq 0) .
\end{aligned}
$$

Let us now analyze a particular eigenvalue problem in four different contexts. Given the Hermitian matrix

$$
M=\left(\begin{array}{cc}
1 & e_{1} \\
-e_{1} & 1
\end{array}\right)
$$

we aim to find its complex, quaternionic and octonionic eigenvalues. In this paper, $e_{1}$ represents the imaginary unit of the complex field, $\mathbb{C}$, and $e_{1}, e_{2}$ and $e_{3}$ the imaginary units of the quaternionic field, $\mathbb{H}$.

(i) The complex eigenvalue problem (CEP),

$$
\mathrm{CEP}: \quad M \Psi=\lambda \Psi, \quad \Psi=\left(\begin{array}{l}
\psi_{a} \\
\psi_{b}
\end{array}\right), \quad \psi_{a, b} \text { and } \lambda \in \mathbb{C},
$$

can be easily solved by standard calculations. Its eigenvectors and eigenvalues are

$$
\left\{\psi_{a}, \psi_{b} ; \lambda\right\}_{1,2}=\left\{z, e_{1} z ; 0\right\}_{1} \text { and }\left\{z,-e_{1} z ; 2\right\}_{2}, z \in \mathbb{C} .
$$

(ii) In exactly the same way, we can establish the quaternionic version of Eq.(6). Due to the non-commutativity of quaternions we have to introduce left and right eigenvalue problems. Let us first discuss the quaternionic left eigenvalue problem (QLEP),

$$
\text { QLEP : } \quad M \Psi=\lambda \Psi, \quad \Psi=\left(\begin{array}{c}
\psi_{a} \\
\psi_{b}
\end{array}\right), \quad \psi_{a, b} \text { and } \lambda \in \mathbb{H} .
$$

After some algebraic manipulations, we find the following eigenvectors and eigenvalues

$$
\left\{\psi_{a}, \psi_{b} ; \lambda\right\}=\left\{q,\left(\alpha e_{1}+\beta e_{2}+\gamma e_{3}\right) q ; 1-\alpha+\beta e_{3}-\gamma e_{2}\right\},
$$

where

$$
\alpha^{2}+\beta^{2}+\gamma^{2}=1, \quad \alpha, \beta, \gamma \in \mathbb{R} \quad \text { and } \quad q \in \mathbb{H} .
$$

In addition to the complex result $(\beta=\gamma=0$ and $q=z)$ we find a surprising result, that is the Hermitian matrix $M$ has quaternionic left eigenvalues. This apparent paradox is soon explained by observing that, due to the non-commutativity of the quaternionic algebra, the standard proof used to show that complex Hermitian matrices have real eigenvalues,

$$
\Psi^{\dagger}(M \Psi)=(M \Psi)^{\dagger} \Psi \Rightarrow \Psi^{\dagger} \lambda \Psi=(\lambda \Psi)^{\dagger} \Psi\left[=\Psi^{\dagger} \lambda^{\dagger} \Psi\right]
$$


fails for quaternions. In fact, due to the position of the quaternionic eigenvalue $\lambda$,

$$
\Psi^{\dagger} \lambda \Psi=\Psi^{\dagger} \lambda^{\dagger} \Psi \Rightarrow \lambda=\lambda^{\dagger} \text {. }
$$

(iii) To overcome this difficulty, we change the position of the eigenvalue in Eq.(17), and introduce the quaternionic right eigenvalues problem (QREP),

$$
\text { QREP : } \quad M \Psi=\Psi \lambda, \quad \Psi=\left(\begin{array}{c}
\psi_{a} \\
\psi_{b}
\end{array}\right), \quad \psi_{a, b} \text { and } \lambda \in \mathbb{H} .
$$

Simple calculations show that the QREP has the same eigenvalues of the CEP, i.e.

$$
\left\{\psi_{a}, \psi_{b} ; \lambda\right\}_{1,2}=\left\{q, e_{1} q ; 0\right\}_{1} \text { and }\left\{q,-e_{1} q ; 2\right\}_{2} .
$$

The position of the quaternionic eigenvalue plays a fundamental role to guarantee real eigenvalues for Hermitian matrices,

$$
\Psi^{\dagger}(M \Psi)=(M \Psi)^{\dagger} \Psi \Rightarrow \Psi^{\dagger} \Psi \lambda=(\Psi \lambda)^{\dagger} \Psi\left[=\lambda^{\dagger} \Psi^{\dagger} \Psi\right] \Rightarrow \lambda=\lambda^{\dagger} .
$$

(iv) What happens for quaternions suggests to consider an octonionic right-eigenvalue problem (OREP),

$$
\text { OREP }: \quad M \Psi=\Psi \lambda, \quad \Psi=\left(\begin{array}{c}
\psi_{a} \\
\psi_{b}
\end{array}\right), \quad \psi_{a, b} \text { and } \lambda \in \mathbb{O} .
$$

The matrix equation (9) implies

$$
-\psi_{b}^{-1}\left(e_{1} \psi_{a}\right)=\psi_{a}^{-1}\left(e_{1} \psi_{b}\right) \text { and } \lambda=1+\psi_{a}^{-1}\left(e_{1} \psi_{b}\right) .
$$

Restricting ourselves to quaternionic sub-algebras containing $e_{1}$, i.e.

$$
e_{1} e_{2} e_{3}, e_{1} e_{4} e_{5} \text { and } e_{1} e_{7} e_{6}
$$

we immediately find the following solutions

$$
\begin{aligned}
\left\{\psi_{a}, \psi_{b} ; \lambda\right\}_{1,2}= & \left\{e_{2}, e_{3} ; 0\right\}_{1} \quad \text { and } \begin{aligned}
& \left\{e_{2},-e_{3} ; 2\right\}_{2}, \\
& \left\{e_{4}, e_{5} ; 0\right\}_{1} \\
& \left\{e_{7}, e_{6} ; 0\right\}_{1}
\end{aligned} \quad\left\{e_{4},-e_{5} ; 2\right\}_{2}, \\
& \left\{e_{7},-e_{6} ; 2\right\}_{2} .
\end{aligned}
$$

To simplify our discussion, in this example we choose the quaternionic sub-algebra $e_{1} e_{2} e_{3}$. It is rather surprising that, notwithstanding the right position of the octonionic eigenvalue, we find, as for the case of the QLEP, new solutions characterized by not real eigenvalues:

$$
\begin{aligned}
& \left\{\psi_{a}, \psi_{b} ; \lambda\right\}_{1,10}=\left\{e_{2}, e_{3} ; 0\right\}_{1} \quad \text { and }\left\{e_{2},-e_{3} ; 2\right\}_{2} \text {, } \\
& \left\{e_{2}, e_{4} ; 1-e_{7}\right\}_{3} \quad\left\{e_{2},-e_{4} ; 1+e_{7}\right\}_{4} \text {, } \\
& \left\{e_{2}, e_{5} ; 1+e_{6}\right\}_{5} \quad\left\{e_{2},-e_{5} ; 1-e_{6}\right\}_{6}, \\
& \left\{e_{2}, e_{6} ; 1-e_{5}\right\}_{7} \quad\left\{e_{2},-e_{6} ; 1+e_{5}\right\}_{8} \text {, } \\
& \left\{e_{2}, e_{7} ; 1+e_{4}\right\}_{9} \quad\left\{e_{2},-e_{7} ; 1-e_{4}\right\}_{10} .
\end{aligned}
$$

This result is well known in literature. For a deeper discussion of similar problems encountered in studying octonionic eigenvalue equations for Hermitian matrices and for mathematical techniques to find the new not real eigenvalues, the best references are [17, 18, 19, 20, 21]. In this paper, we are interested in presenting a conversion 
method, in the main quite practical, to find real eigenvalues of octonionic operators. The matrix representations for these operators [22, 23] could be very useful in view of possible applications in quantum mechanics. Before to elaborate the core of our article, let us briefly explain where the proof given for the CEP and for the QREP is lacking in validity. Due the non-associativity of the octonionic algebra

$$
\Psi^{\dagger}(M \Psi) \neq(M \Psi)^{\dagger} \Psi\left[=\left(\Psi^{\dagger} M\right) \Psi\right] .
$$

Consequently, the position of the octonionic eigenvalue in the OREP is not sufficient to guarantee the real nature of Hermitian matrix eigenvalues.

The results of this preliminary study are instructive for several reasons. In the first place, they show that using a quaternionic algebra a great simplification can be obtained by making an initial assumption concerning the position of the quaternionic eigenvalue. Secondly, the possibility to apply a similarity transformation $(u \lambda \bar{u}=z)$ opens the door to translate the QREP in its complex or real counterpart. This method is frequently employed to circumvent the difficulties attendant the non-commutativity of quaternions [24]. What we aim to prove in this paper is a stronger result. The existence of conversion rules for octonionic operators allow to give a practical method to solve the coupled octonionic eigenvalue problem (COEP) which reduces to the QREP in the quaternionic limit [1].

\section{Octonionic eigenvalue problem}

\subsection{Real matrix conversion}

The non-associativity of octonions seems to suggest the impossibility to obtain a real matrix representations (with the standard matrix multiplication rules) for octonionic operators [23]. Nevertheless, the use of left/right barred octonionic operators allow to reproduce the $G L(8, \mathbb{R})$ group $[22$. For the convenience of the reader and to make our exposition as self-contained as possible, we repeat part of the material exposed in ref. [22].

It's well known that octonions are a non commutative algebra so we must distinguish between left and right actions of the octonionic imaginary units $e_{m}$, by introducing operators $L_{o_{1}}$ and $R_{o_{2}}$ whose action on octonionic functions of a real variable, $\psi$, $\psi: \mathbb{R} \rightarrow \mathbb{O}$, gives

$$
L_{o_{1}} \psi=o_{1} \psi \quad \text { and } \quad R_{o_{2}} \psi=\psi o_{2},
$$

But, octonions are also a non associative algebra that means

$$
o_{1}\left(\psi o_{2}\right) \neq\left(o_{1} \psi\right) o_{2}
$$

so $L_{o_{1}} R_{O_{2}} \psi$ must be calculated, necessarily, in the order the operations appear, that is,

$$
L_{o_{1}} R_{o_{2}} \psi=L_{o_{1}}\left(R_{o_{2}} \psi\right)=o_{1}\left(\psi o_{2}\right)
$$

and

$$
R_{o_{2}} L_{o_{1}} \psi=R_{o_{2}}\left(L_{o_{1}} \psi\right)=\left(o_{1} \psi\right) o_{2}
$$


Naturally the same holds for

$$
L_{o_{1}} L_{o_{2}} \psi=L_{o_{1}}\left(L_{o_{2}} \psi\right)=o_{1}\left(o_{2} \psi\right)
$$

and

$$
R_{o_{1}} R_{o_{2}} \psi=R_{o_{1}}\left(R_{o_{2}} \psi\right)=R_{o_{1}}\left(\psi o_{2}\right)=\left(\psi o_{2}\right) o_{1}
$$

In order to write the more general octonionic operator it's enough to describe all the possible actions of the imaginary units $e_{m}$. So, computing the operators just described for the imaginary units we find

$$
\begin{array}{cr}
1, L_{m}, R_{m} & 15 \text { elements } \\
L_{m} R_{m}=R_{m} L_{m} & 7 \text { elements } \\
L_{m} R_{n} m \neq n & 42 \text { elements } \\
R_{n} L_{m} m \neq n & 42 \text { elements }
\end{array}
$$

for $m, n=1, \cdots, 7$, which totalizes 106 operators. However, it is possible to prove that $L_{m} R_{n}$, can be expressed by a suitable combination of $R_{n} L_{m}$ operators, reducing to 64 the previous 106 elements [22]. For example, we have

$$
L_{m} R_{n}+L_{n} R_{m}=R_{n} L_{m}+R_{m} L_{n} .
$$

As explicitly shown in Appendix A of ref. [22], it is possible to prove the linear independence of these 64 elements which represent the most general octonionic operator

$$
o_{0}+\sum_{m=1}^{7} R_{m} L_{o_{m}}
$$

with $o_{0}, \cdots, o_{7} \in \mathbb{O}$. This shows the correspondence between our generalized octonions, equation (12), and $G L(8, \mathbb{R})$.

In paper [23], the authors give representations of octonions and other nonassociative algebras by special matrices, which are endowed with special multiplication rules. The introduction of left/right octonionic operators allow us to establish the isomorphism between themselves and $G L(8, \mathbb{R})$ with the standard multiplication rules. In order to explain the idea of conversion, let us look explicitly at the action of the operators $R_{1}$ and $L_{2}$ on a generic octonionic function $\psi: \mathbb{R} \rightarrow \mathbb{O}$ :

$$
\psi(x)=\psi_{0}(x)+e_{1} \psi_{1}(x)+e_{2} \psi_{2}(x)+e_{3} \psi_{3}(x)+e_{4} \psi_{4}(x)+e_{5} \psi_{5}(x)+e_{6} \psi_{6}(x)+e_{7} \psi_{7}(x)
$$

with $\psi_{0, \cdots, 7}: \mathbb{R} \rightarrow \mathbb{R}$. In order to simplify our notation we omit $x$ variable. So, let us calculate $R_{1} \psi$ that gives

$$
R_{1} \psi=\psi e_{1}=e_{1} \psi_{0}-\psi_{1}-e_{3} \psi_{2}+e_{2} \psi_{3}-e_{5} \psi_{4}+e_{4} \psi_{5}+e_{7} \psi_{6}-e_{6} \psi_{7}
$$

and now, we calculate

$$
L_{2} \psi=e_{2} \psi=e_{2} \psi_{0}-e_{3} \psi_{1}-\psi_{2}+e_{1} \psi_{3}+e_{6} \psi_{4}+e_{7} \psi_{5}-e_{4} \psi_{6}-e_{5} \psi_{7}
$$


If we represent the octonionic function $\psi$ by a real column vector $8 \times 1$

$$
\psi \leftrightarrow \Psi=\left(\begin{array}{c}
\psi_{0} \\
\psi_{1} \\
\psi_{2} \\
\psi_{3} \\
\psi_{4} \\
\psi_{5} \\
\psi_{6} \\
\psi_{7}
\end{array}\right)
$$

we can rewrite $\psi e_{1}$ and $e_{2} \psi$ respectively as

$$
\left(\begin{array}{rrrrrrrr}
0 & -1 & 0 & 0 & 0 & 0 & 0 & 0 \\
1 & 0 & 0 & 0 & 0 & 0 & 0 & 0 \\
0 & 0 & 0 & 1 & 0 & 0 & 0 & 0 \\
0 & 0 & -1 & 0 & 0 & 0 & 0 & 0 \\
0 & 0 & 0 & 0 & 0 & 1 & 0 & 0 \\
0 & 0 & 0 & 0 & -1 & 0 & 0 & 0 \\
1 & 0 & 0 & 0 & 0 & 0 & 0 & -1 \\
1 & 0 & 0 & 0 & 0 & 0 & 1 & 0
\end{array}\right)\left(\begin{array}{l}
\psi_{0} \\
\psi_{1} \\
\psi_{2} \\
\psi_{3} \\
\psi_{4} \\
\psi_{5} \\
\psi_{6} \\
\psi_{7}
\end{array}\right)=\left(\begin{array}{r}
-\psi_{1} \\
\psi_{0} \\
\psi_{3} \\
-\psi_{2} \\
\psi_{5} \\
-\psi_{4} \\
-\psi_{7} \\
\psi_{6}
\end{array}\right)
$$

and

$$
\left(\begin{array}{rrrrrrrr}
0 & 0 & -1 & 0 & 0 & 0 & 0 & 0 \\
0 & 0 & 0 & 1 & 0 & 0 & 0 & 0 \\
1 & 0 & 0 & 0 & 0 & 0 & 0 & 0 \\
0 & -1 & 0 & 0 & 0 & 0 & 0 & 0 \\
0 & 0 & 0 & 0 & 0 & 0 & -1 & 0 \\
0 & 0 & 0 & 0 & 0 & 0 & 0 & -1 \\
0 & 0 & 0 & 0 & 1 & 0 & 0 & 0 \\
0 & 0 & 0 & 0 & 0 & 1 & 0 & 0
\end{array}\right)\left(\begin{array}{c}
\psi_{0} \\
\psi_{1} \\
\psi_{2} \\
\psi_{3} \\
\psi_{4} \\
\psi_{5} \\
\psi_{6} \\
\psi_{7}
\end{array}\right)=\left(\begin{array}{r}
-\psi_{2} \\
\psi_{3} \\
\psi_{0} \\
-\psi_{1} \\
-\psi_{6} \\
-\psi_{7} \\
\psi_{4} \\
\psi_{5}
\end{array}\right)
$$

Following this procedure, we can construct the complete set of conversion rules for the imaginary units operators $L_{m}$ and $R_{m}$. Observe that if we multiply the matrices that represent $L_{1}$ and $L_{2}$ we will find a new matrix that is different from $L_{3}$, that is, $L_{1} L_{2} \neq L_{3}$ while $e_{1} e_{2}=e_{3}$. This bluff is soon explained. In deducing our conversion rules, we understand octonions as operators, and so they must be applied to a certain octonionic function, $\psi$. If we have the octonionic relation

$$
\left(e_{1} e_{2}\right) \psi=e_{3} \psi
$$

the matrix counterpart will be

$$
L_{3} \psi
$$

since the matrix counterparts are defined by their action upon a function and not upon another operator. On the other hand,

$$
e_{1}\left(e_{2} \psi\right) \neq e_{3} \psi
$$


will be translated by

$$
L_{1} L_{2} \psi=L_{1}\left(L_{2} \psi\right) \neq L_{3} \psi
$$

We have to differentiate between two kinds of multiplication, one, for octonions, denoted by a middle dot, ".", and the other, for octonionic operators, denoted by the usual multiplication sign " $\times$ ". At the level of octonions, one has

$$
e_{1} \cdot e_{2}=e_{3}
$$

but at the level of octonionic operators

$$
L_{1} \times L_{2} \neq L_{3}
$$

but

$$
L_{1} \times L_{2}=L_{3}+R_{2} L_{1}-L_{1} R_{2}
$$

Observe the matricial representations of our operators enable us to reproduce the octonion nonassociativity by the matrix algebra. Consider, for example,

$$
\begin{aligned}
& R_{1} L_{3} \psi=R_{1}\left(L_{3} \psi\right)=\left(e_{3} \psi\right) e_{1}= \\
& e_{2} \psi_{0}-e_{3} \psi_{1}+\psi_{2}-e_{1} \psi_{3}-e_{6} \psi_{4}-e_{7} \psi_{5}+e_{4} \psi_{6}+e_{5} \psi_{7}
\end{aligned}
$$

which gives, in matricial representation

$$
\left(\begin{array}{rrrrrrrr}
0 & 0 & 1 & 0 & 0 & 0 & 0 & 0 \\
0 & 0 & 0 & -1 & 0 & 0 & 0 & 0 \\
1 & 0 & 0 & 0 & 0 & 0 & 0 & 0 \\
0 & -1 & 0 & 0 & 0 & 0 & 0 & 0 \\
0 & 0 & 0 & 0 & 0 & 0 & 1 & 0 \\
0 & 0 & 0 & 0 & 0 & 0 & 0 & 1 \\
0 & 0 & 0 & 0 & -1 & 0 & 0 & 0 \\
0 & 0 & 0 & 0 & 0 & -1 & 0 & 0
\end{array}\right)\left(\begin{array}{l}
\psi_{0} \\
\psi_{1} \\
\psi_{2} \\
\psi_{3} \\
\psi_{4} \\
\psi_{5} \\
\psi_{6} \\
\psi_{7}
\end{array}\right)=\left(\begin{array}{r}
\psi_{2} \\
-\psi_{3} \\
\psi_{0} \\
-\psi_{1} \\
\psi_{6} \\
\psi_{7} \\
-\psi_{4} \\
-\psi_{5}
\end{array}\right)
$$

whereas

$$
\begin{aligned}
& L_{3} R_{1} \psi=L_{3}\left(R_{1} \psi\right)=e_{3}\left(\psi e_{1}\right)= \\
& e_{2} \psi_{0}-e_{3} \psi_{1}+\psi_{2}-e_{1} \psi_{3}+e_{6} \psi_{4}+e_{7} \psi_{5}-e_{4} \psi_{6}-e_{5} \psi_{7}
\end{aligned}
$$

which gives, in matricial representation

$$
\left(\begin{array}{rrrrrrrr}
0 & 0 & 1 & 0 & 0 & 0 & 0 & 0 \\
0 & 0 & 0 & -1 & 0 & 0 & 0 & 0 \\
1 & 0 & 0 & 0 & 0 & 0 & 0 & 0 \\
0 & -1 & 0 & 0 & 0 & 0 & 0 & 0 \\
0 & 0 & 0 & 0 & 0 & 0 & -1 & 0 \\
0 & 0 & 0 & 0 & 0 & 0 & 0 & -1 \\
0 & 0 & 0 & 0 & 1 & 0 & 0 & 0 \\
0 & 0 & 0 & 0 & 0 & 1 & 0 & 0
\end{array}\right)\left(\begin{array}{l}
\psi_{0} \\
\psi_{1} \\
\psi_{2} \\
\psi_{3} \\
\psi_{4} \\
\psi_{5} \\
\psi_{6} \\
\psi_{7}
\end{array}\right)=\left(\begin{array}{r}
\psi_{2} \\
-\psi_{3} \\
\psi_{0} \\
-\psi_{1} \\
-\psi_{6} \\
-\psi_{7} \\
\psi_{4} \\
\psi_{5}
\end{array}\right)
$$


From the 106 elements given in (11), that we can rewrite in the matricial form, we can extract two different basis bases for $G L(8, \mathbb{R})$ those are

$$
1, L_{m}, R_{n}, R_{n} L_{m}
$$

or

$$
1, L_{m}, R_{n}, L_{m} R_{n}
$$

We now remark some difficulties deriving from octonion nonassociativity. When we translate from generalized octonions to $8 \times 8$ real matrices there is no problem. For example, in the octonionic object

$$
e_{4}\left\{\left[\left(e_{6} \psi\right) e_{1}\right] e_{5}\right\}
$$

we quickly recognize the operators

$$
L_{4} R_{5} \quad \text { and } \quad R_{1} L_{6} .
$$

Thus, rewriting the previous object we have

$$
L_{4} R_{5} R_{1} L_{6} \psi
$$

In going from $8 \times 8$ real matrices to octonions, we should be careful in ordering. For example,

$A B \psi$

can be understood as

$$
(A B) \psi \quad \text { or } \quad A(B \psi) \text {. }
$$

For example, by choosing $A=L_{1}$ and $B=L_{2}$, we have two possible different translations

$$
\left(L_{1} L_{2}\right) \psi \rightarrow\left(e_{1} e_{2}\right) \psi=e_{3} \psi \quad \text { or } \quad L_{1}\left(L_{2} \psi\right) \rightarrow e_{1}\left(e_{2} \psi\right)
$$

Which is the right equation? The second translation is the right one, observe that $L_{1} L_{2} \neq L_{3}$. So, when we find multiple multiplications, to translate correctly in the octonionic formalism, we have to use the following ordering rule

$$
A B C \ldots Z \psi=A(B(\ldots(Z \psi))) \text {. }
$$

\subsection{Coupled eigenvalue problem}

Due to the non commutativity of quaternions, they have represented a challenge when mathematicians tried to extend the well-known complex eigenvalue problem to the quaternionic field. The eigenvalue problem for $\mathbb{H}$-linear quaternionic operators, which means that only the left action of quaternionic imaginary units are present, and an extension to $\mathbb{C}$ and $\mathbb{R}$-linear quaternionic operator that are, respectively, operators which contain, besides the left action of all units, the right action of only one imaginary unit and the right action of all quaternionic imaginary units, has been recently discussed and

can be found in [1, 2]. The quaternionic eigenvalue problem allowed the first steps in the theory of quaternionic differential equations [3] and, the latter, made possible 
the study of the Schrödinger equation in the presence of a quaternionic potential in view of analyze the quaternionic tunnelling effect [12]. It's worth pointing out that the eigenvalue problem for $\mathbb{R}$-linear quaternionic operators provides a remarkable version of the eigenvalue problem dividing it in two coupled equations. For the octonionic eigenvalue problem we resort to this same method that gives out a coupled eigenvalue problem.

Consider the octonionic real eigenvalue problem

$$
\mathcal{O} \psi=\psi r, \quad r \in \mathbb{R}
$$

which matricial conversion is given by

$$
M_{o} \Psi_{8 \times 1}=r \Psi_{8 \times 1},
$$

where $M_{o}$ is a real square matrix of order 8 . Note that the eigenvalue must be real and this condition is too restrictive. The matrix $M_{o} \in M_{8}[\mathbb{R}]$ will probably have complex eigenvalues and, consequently, complex eigenvectors but when this occur the conversion backward to the octonionic formalism is not possible. This is exactly the same problem when studying the eigenvalue problem for real linear quaternionic operators. For a deeper discussion of this subject we refer the reader to [2]. We will use the same technique to study the octonionic eigenvalue problem but here this is done in a much more roughly way. In order to differentiate between octonionic and complex formalisms, after the conversion from octonion to matrices, the complex unit will be denoted by $i$. So, accepting that $M_{o}$ has complex eigenvalues we can rewrite the eigenvalue problem (16) as

$$
M_{o} \Psi=z \Psi, \quad z \in \mathbb{C} .
$$

Introducing $\Psi=\xi+i \eta$, where $\xi$ and $\eta$ are real column vectors $8 \times 1$ and $z=a+i b$, with $a, b \in \mathbb{R}$ in (17) we have

$$
M_{o}(\xi+i \eta)=(a+i b)(\xi+i \eta)
$$

Separating the real from the imaginary part we have a coupled equation given by

$$
\begin{aligned}
& M_{o} \xi=a \xi-b \eta \\
& M_{o} \eta=a \eta+b \xi
\end{aligned}
$$

Now, all the elements present in (18) are real which allows the conversion to the octonionic formalism. Let see a one dimensional example. Consider the problem

$$
e_{4} \psi=\psi \lambda \text {. }
$$

We want to find $\xi, \eta$ and $\lambda \in \mathbb{C}$ that satisfies (18). The first step is to translate the 
problem in the matricial form which is given by

$$
\left(\begin{array}{rrrrrrrr}
0 & 0 & 0 & 0 & -1 & 0 & 0 & 0 \\
0 & 0 & 0 & 0 & 0 & 1 & 0 & 0 \\
0 & 0 & 0 & 0 & 0 & 0 & 1 & 0 \\
0 & 0 & 0 & 0 & 0 & 0 & 0 & 1 \\
1 & 0 & 0 & 0 & 0 & 0 & 0 & 0 \\
0 & -1 & 0 & 0 & 0 & 0 & 0 & 0 \\
0 & 0 & -1 & 0 & 0 & 0 & 0 & 0 \\
0 & 0 & 0 & -1 & 0 & 0 & 0 & 0
\end{array}\right)\left(\begin{array}{l}
\psi_{0} \\
\psi_{1} \\
\psi_{2} \\
\psi_{3} \\
\psi_{4} \\
\psi_{5} \\
\psi_{6} \\
\psi_{7}
\end{array}\right)=z\left(\begin{array}{l}
\psi_{0} \\
\psi_{1} \\
\psi_{2} \\
\psi_{3} \\
\psi_{4} \\
\psi_{5} \\
\psi_{6} \\
\psi_{7}
\end{array}\right) .
$$

The eigenvalues and eigenvectors of the problem (19) are, respectively,

eigenvalues:

eigenvectors:

$$
\begin{array}{llllllll}
-i & -i & -i & -i & i & i & i & i
\end{array}
$$

$$
\left(\begin{array}{l}
0 \\
0 \\
0 \\
i \\
0 \\
0 \\
0 \\
1
\end{array}\right)\left(\begin{array}{l}
0 \\
0 \\
i \\
0 \\
0 \\
0 \\
1 \\
0
\end{array}\right) \quad\left(\begin{array}{l}
0 \\
i \\
0 \\
0 \\
0 \\
1 \\
0 \\
0
\end{array}\right) \quad\left(\begin{array}{r}
-i \\
0 \\
0 \\
0 \\
1 \\
0 \\
0 \\
0
\end{array}\right) \quad\left(\begin{array}{r}
0 \\
0 \\
0 \\
-i \\
0 \\
0 \\
0 \\
1
\end{array}\right) \quad\left(\begin{array}{r}
0 \\
0 \\
-i \\
0 \\
0 \\
0 \\
1 \\
0
\end{array}\right) \quad\left(\begin{array}{r}
0 \\
-i \\
0 \\
0 \\
0 \\
1 \\
0 \\
0
\end{array}\right)\left(\begin{array}{l}
i \\
0 \\
0 \\
0 \\
1 \\
0 \\
0 \\
0
\end{array}\right)
$$

Consider $\lambda=-i$ and the respective eigenvector $\Psi=(0,0,0, i, 0,0,0,1)^{t}$. We have $a=0$ and $b=-1$ and separating the real from the imaginary part of the eigenvector,

$$
\left(\begin{array}{l}
0 \\
0 \\
0 \\
i \\
0 \\
0 \\
0 \\
1
\end{array}\right)=\left(\begin{array}{l}
0 \\
0 \\
0 \\
0 \\
0 \\
0 \\
0 \\
1
\end{array}\right)+i\left(\begin{array}{l}
0 \\
0 \\
0 \\
1 \\
0 \\
0 \\
0 \\
0
\end{array}\right)
$$


The octonionic eigenvalue problem

we obtain

$$
\xi=\left(\begin{array}{c}
0 \\
0 \\
0 \\
0 \\
0 \\
0 \\
0 \\
1
\end{array}\right) \quad \text { and } \eta=\left(\begin{array}{c}
0 \\
0 \\
0 \\
1 \\
0 \\
0 \\
0 \\
0
\end{array}\right)
$$

Note that all elements $a, b, \xi$ and $\eta$ are real so we can transform each of them in octonionic numbers. This procedure gives

$$
\xi=e_{7} \quad \text { and } \quad \eta=e_{3}
$$

and the coupled equations, already in the octonionic formalism, became

$$
\begin{aligned}
& e_{4}\left(e_{7}\right)=0 e_{3}+1 e_{3}=e_{3} \\
& e_{4}\left(e_{3}\right)=-e_{7}+0 e_{3}=-e_{7}
\end{aligned}
$$

Following the same procedure, consider the $2 \times 2$ octonionic matrix

$$
\left(\begin{array}{ll}
1 & e_{4} \\
0 & e_{5}
\end{array}\right)
$$

Its conversion lead us to a $16 \times 16$ real matrix which eigenvalues are $i,-i$ and 1 . The algebraic multiplicity of $\lambda$ is equal to its geometric multiplicity. In this case $i$ and $-i$ have algebraic multiplicity equal 4 and 1 has algebraic multiplicity equal 8 . By taking the eigenvalue $-i$ we have $a=0$ and $b=-1$ and the correspondent real vectors $(\xi$ and $\eta)$ are given by $\xi=(0,0,0,-1,0,0,1,0,0,0,0,0,0,0,0,2)^{t}$ and $\eta=(0,0,0,-1,0,0,1,0,0,0,2,0,0,0,0,0)^{t}$ which, in octonionic formalism are written as

$$
\xi=\left(\begin{array}{c}
-e_{3}+e_{6} \\
2 e_{7}
\end{array}\right) \quad \text { and } \quad \eta=\left(\begin{array}{c}
e_{3}+e_{6} \\
2 e_{2}
\end{array}\right)
$$

and the coupled equation became

$$
\begin{aligned}
& \left(\begin{array}{ll}
1 & e_{4} \\
0 & e_{5}
\end{array}\right)\left(\begin{array}{c}
-e_{3}+e_{6} \\
2 e_{7}
\end{array}\right)=0\left(\begin{array}{c}
-e_{3}+e_{6} \\
2 e_{7}
\end{array}\right)-(-1)\left(\begin{array}{c}
e_{3}+e_{6} \\
2 e_{2}
\end{array}\right) \\
& \left(\begin{array}{ll}
1 & e_{4} \\
0 & e_{5}
\end{array}\right)\left(\begin{array}{c}
e_{3}+e_{6} \\
2 e_{2}
\end{array}\right)=-\left(\begin{array}{c}
-e_{3}+e_{6} \\
2 e_{7}
\end{array}\right)+0\left(\begin{array}{c}
e_{3}+e_{6} \\
2 e_{2}
\end{array}\right)
\end{aligned}
$$

It's interesting to note that when the eigenvalue problem is considered for a $\mathbb{H}$-linear quaternionic operator, we have

$$
\mathcal{Q} \psi=\psi \lambda=\psi a+\psi i b .
$$

Multiplying the expression above by $i$ we obtain

$$
(\mathcal{Q} \psi) i=(\psi a) i+(\psi i b) i
$$


but quaternions are associative which means that

$$
\mathcal{Q}(\psi i)=(\psi i) a-\psi b .
$$

The eigenvectors $\psi i$ and $\psi$ play the role of $\xi$ and $\eta$, respectively, in the coupled equations. Thus,

$$
\begin{aligned}
& \mathcal{Q} \xi=\mathcal{Q}(\psi i)=(\psi i) a-\psi b=\xi a-\eta b \\
& \mathcal{Q} \eta=\mathcal{Q} \psi=\psi a+(\psi i) b=\eta a+\xi b
\end{aligned}
$$

which means that it's possible to associate to any pair of functions $\xi$ and $\eta$ that solves the system (21) a corresponding eigenvector of (20).

\subsection{Complexified eigenvalue problem}

An alternative way to solve octonionic eigenvalue problems is to consider complexified octonions, $\mathbb{C}(1, i) \times \mathbb{O}$, which allow to immediately translate Eq. (17) as follows

$$
\mathcal{O} \Phi=\Phi z
$$

where $\mathcal{O}$ is a octonionic matrix, $\Psi \in \mathbb{C}(1, i) \times \mathbb{O}$ and $z \in \mathbb{C}(1, i)$. Introducing $\Phi=\phi_{1}+i \phi_{2}$ and $z=a+i b$ we have

$$
\mathcal{O}\left(\phi_{1}+i \phi_{2}\right)=\left(\phi_{1}+i \phi_{2}\right)(a+i b), \quad \mathcal{O}, \phi_{1}, \phi_{2} \in \mathbb{O}, \quad a, b \in \mathbb{R} .
$$

Algebraic manipulations lead us to the solution of the problem. For example, consider the same problem presente in Section 2.2 , which is

$$
e_{4}\left(\phi_{1}+i \phi_{2}\right)=\left(\phi_{1}+i \phi_{2}\right)(a+i b) .
$$

Now, multiplying (23) by $e_{4}$ from the left we find

$$
e_{4}\left[e_{4}\left(\phi_{1}+i \phi_{2}\right)\right]=e_{4}\left[\left(\phi_{1}+i \phi_{2}\right)(a+i b)\right],
$$

which gives

$$
-\left(\phi_{1}+i \phi_{2}\right)=\left[e_{4}\left(\phi_{1}+i \phi_{2}\right)\right](a+i b)=(a+i b)^{2}\left(\phi_{1}+i \phi_{2}\right),
$$

since $e_{4}$ commutes with $a+i b$. Equation above gives the very simple system

$$
a^{2}-b^{2}=-1 \quad \text { and } \quad 2 a b=0
$$

which solution is $a=0$ and $b= \pm 1$. So, the eigenvalue is $\pm i$. Now, reintroducing $-i$ in the eigenvalue equation we find the eigenvectors. Then, for $z=-i$ we have

$$
e_{4}\left(\phi_{1}+i \phi_{2}\right)=\left(\phi_{1}+i \phi_{2}\right)(-i)=-i \phi_{1}+\phi_{2} \quad \Rightarrow \quad e_{4} \phi_{1}=\phi_{2}, e_{4} \phi_{2}=-\phi_{1}
$$

Remembering that the octonionic imaginary units obey the relation

$$
123,145,176,246,257,347 \text { and } 365,
$$


it's easy to see that $\phi_{1}$ can assume the values $e_{4}, e_{5}, e_{6}$ and $e_{7}$ and $\phi_{2}$ can assume, respectively, the values $-1, e_{1}, e_{2}$ and $e_{3}$. Thus

$$
\begin{aligned}
& e_{4}\left(e_{4}-i\right)=\left(e_{4}-i\right)(-i), \\
& e_{4}\left(e_{5}+e_{1}\right)=\left(e_{5}+e_{1}\right)(-i), \\
& e_{4}\left(e_{6}+e_{2}\right)=\left(e_{6}+e_{2}\right)(-i), \\
& e_{4}\left(e_{7}+e_{3}\right)=\left(e_{7}+e_{3}\right)(-i) .
\end{aligned}
$$

Let us consider the complexified octonion formalism to solve another example from section 2.2. Consider

$$
\left(\begin{array}{ll}
1 & e_{4} \\
0 & e_{5}
\end{array}\right)
$$

We want to find the eigenvalues $\lambda=a+i b, a, b \in \mathbb{R}$ and the respective eigenvectors which have the following form

$$
(\phi)_{1} \phi_{2}+i(\psi)_{1} \psi_{2}, \quad \phi_{1,2}, \psi_{1,2} \in \mathbb{O} \text {. }
$$

The eigenvalue problem is

$$
\left(\begin{array}{ll}
1 & e_{4} \\
0 & e_{5}
\end{array}\right)(\phi)_{1}+i \psi_{1} \phi_{2}+i \psi_{2}=(\phi)_{1}+i \psi_{1} \phi_{2}+i \psi_{2}(a+i b)
$$

Performing the matrix multiplication, we have

$$
\begin{aligned}
& e_{4}\left(\phi_{2}+i \psi_{2}\right)=\left(\phi_{1}+i \psi_{1}\right)(a-1+i b), \\
& e_{5}\left(\phi_{2}+i \psi_{2}\right)=\left(\phi_{2}+i \psi_{2}\right)(a+i b)
\end{aligned}
$$

The proceedure to solve eq.(26) is almost the same as the previously just done. So, repeating it and fixing the eigenvalue $-i$ we find

$$
\phi_{2}+i \psi_{2} \in\left\{1+i e_{5}, e_{1}+i e_{4}, e_{3}+i e_{6}, e_{7}+i e_{2}\right\}
$$

In order to find $\phi_{1}$ and $\psi_{1}$ we have to fix an eigenvalue on the set above which introduced in eq.(25) gives the result. To find all the possibilities we have to do this for all elements of the set. To illustrate this, we choose $1+i e_{5}$. Remenber that we are working with $\lambda=-i$, so

$$
e_{4}\left(1+i e_{5}\right)=\left(\phi_{1}+i \psi_{1}\right)(-1-i) \Rightarrow e_{4}+i e_{1}=-\phi_{1}-i \phi_{1}-i \psi_{1}+\psi_{1}
$$

Solving the system of equations we find $\phi_{1}=-\left(e_{1}+e_{4}\right) / 2$ and $\psi_{1}=\left(e_{4}-e_{1}\right) / 2$ and we have

$$
\left(\begin{array}{ll}
1 & e_{4} \\
0 & e_{5}
\end{array}\right)\left[\left(\begin{array}{c}
-e_{1}-e_{4} \\
2
\end{array}\right)+i\left(\begin{array}{c}
e_{4}-e_{1} \\
2 e_{5}
\end{array}\right)\right]=\left[\left(\begin{array}{c}
-e_{1}-e_{4} \\
2
\end{array}\right)+i\left(\begin{array}{c}
e_{4}-e_{1} \\
2 e_{5}
\end{array}\right)\right](-i) .
$$

Repeating the procedure for the remaining three cases, always for $\lambda=-i$ we have 
The octonionic eigenvalue problem

$$
\begin{aligned}
& \left(\begin{array}{ll}
1 & e_{4} \\
0 & e_{5}
\end{array}\right)\left[\left(\begin{array}{c}
1+e_{5} \\
2 e_{1}
\end{array}\right)+i\left(\begin{array}{c}
1-e_{5} \\
2 e_{4}
\end{array}\right)\right]=\left[\left(\begin{array}{c}
1+e_{5} \\
2 e_{1}
\end{array}\right)+i\left(\begin{array}{c}
1-e_{5} \\
2 e_{4}
\end{array}\right)\right](-i), \\
& \left(\begin{array}{ll}
1 & e_{4} \\
0 & e_{5}
\end{array}\right)\left[\left(\begin{array}{c}
e_{2}-e_{7} \\
-2 e_{3}
\end{array}\right)+i\left(\begin{array}{c}
e_{2}+e_{7} \\
-2 e_{6}
\end{array}\right)\right]=\left[\left(\begin{array}{c}
e_{2}-e_{7} \\
-2 e_{3}
\end{array}\right)+i\left(\begin{array}{c}
e_{2}+e_{7} \\
-2 e_{6}
\end{array}\right)\right](-i), \\
& \left(\begin{array}{ll}
1 & e_{4} \\
0 & e_{5}
\end{array}\right)\left[\left(\begin{array}{c}
e_{6}-e_{3} \\
2 e_{7}
\end{array}\right)+i\left(\begin{array}{c}
e_{3}+e_{6} \\
2 e_{2}
\end{array}\right)\right]=\left[\left(\begin{array}{c}
e_{6}-e_{3} \\
2 e_{7}
\end{array}\right)+i\left(\begin{array}{c}
e_{3}+e_{6} \\
2 e_{2}
\end{array}\right)\right](-i) .
\end{aligned}
$$

Observe that what is missing when comparing the procedure, for a matrix of order 2, just presented with the previous case is that, in addition, from eq.(26) we have

$$
\phi_{2}+i \psi_{2}=0 \quad \Rightarrow \quad \phi_{2}=\psi_{2}=0 \text {. }
$$

Introducing $\phi_{2}+i \psi_{2}=0$ in eq.(25) we obtain

$$
\left(\phi_{1}+i \psi_{1}\right)(a-1+i b)=0 \Rightarrow a=1, b=0 .
$$

So, another eigenvalue of the octonionic problem discussed is $\lambda=1$ which eigenvectors are

$$
\phi_{1}+i \psi_{1}, \forall \phi_{1}, \psi_{1} \in \mathbb{O} \text {. }
$$

\subsection{The group $G L(8, \mathbb{C})$}

In the previous subsections, we have presented two equivalent method to solve the octonionic eigenvalue problem. The translation of octonionic left/right operators by $8 \times 8$ real matrices, discussed in subsection 2.1, gives us the possibility to introduce a coupled eigenvalue equation characterized by two real numbers $a$ and $b$ and two real eigenvectors $\xi$ and $\eta$, see Eq. (18). This real coupled eigenvalue problem can be then translated by using the equivalence between the group $G L(8, \mathbb{R})$ and real linear octonionic operators in a coupled real eigenvalue problem for octonionic operators,

$$
\begin{aligned}
& \mathcal{O}_{\mathbb{R}} \psi_{\xi}=a \psi_{\xi}-b \psi_{\eta}, \\
& \mathcal{O}_{\mathbb{R}} \psi_{\eta}=a \psi_{\eta}+b \psi_{\xi},
\end{aligned}
$$

with $\psi_{\xi}$ and $\psi_{\eta} \in \mathbb{O}, a$ and $b \in \mathbb{R}$ and $\mathcal{O}_{\mathbb{R}}$ represented by a real linear left/right octonionic operator. The presence of two real coupled eigenvalues suggests, see subsection 2.3, the possibility to re-write such a coupled real eigenvalue problem as a "complex" eigenvalue problem. Indeed, by introducing a new complex imaginary unit $i$ which commutes with the imaginary units of the octonionic field $e_{1,2, \ldots, 7}$, we find

$$
\mathcal{O}(\underbrace{\psi_{\xi}+i \psi_{\eta}}_{\Psi \in \mathbb{O} \times \mathbb{C}})=(\underbrace{a+i b}_{z \in \mathbb{C}})\left(\psi_{\xi}+i \psi_{\eta}\right) \text {. }
$$

The introduction of this complex imaginary unit also suggests to extend the matrix translation for real linear octonionic operators,

$$
\mathcal{O}_{\mathbb{R}} \leftrightarrow G L(8, \mathbb{R})
$$


introduced in ref.[22] e re-presented in subsection 2.1, to complex linear octonionic operators

$$
\mathcal{O}_{\mathbb{C}} \leftrightarrow G L(8, \mathbb{C})
$$

Thus, the two equivalent methods to solve the eigenvalue problem for a real linear octonionic operators, presented in subsections 2.2 and 2.3, can be used to solve the eigenvalue problem for complex linear octonionic operators. Observing that a complex linear octonionic operator, $\mathcal{O}_{\mathbb{C}}$, is characterized by two real linear octonionic operators ( $\mathcal{O}_{1 \mathbb{R}}$ and $\mathcal{O}_{2 \mathbb{R}}$ - real and complex part of the complex linear octonionic operator $\left.\mathcal{O}_{\mathbb{C}}\right)$ we can use the matrix translation $\left(\mathcal{O}_{1 \mathbb{R}} \rightarrow M_{1}\right.$ and $\left.\mathcal{O}_{2 \mathbb{R}} \rightarrow M_{2}\right)$ to represent the complex linear operator by the $8 \times 8$ complex matrix $M:=M_{1}+i M_{2}$. By solving the eigenvalue problem for the complex matrix $M$ we find its eigenvectors $\psi \in \mathbb{C}_{8 \times 1}$ and eigenvalues $z \in \mathbb{C}$. Then, we translate back to the octonionic formalism

$$
\mathcal{O}_{\mathbb{C}} \Psi=z \Psi,
$$

with $\Psi \in \mathbb{O} \times \mathbb{C}$ and $z \in \mathbb{C}$. After a discussion of the hermicity of octonionic operators and the appropriate use of scalar product to define probability amplitudes, we shall give an explicit example of a physical problem where this translation can be applied.

\section{On the hermiticity of octonionic matrices and operators}

An important step towards a generalization of standard quantum theories is the use of complex scalar products or complex geometry as referred to by Rembieliński [25].

In quantum mechanics, it's well known that an anti-Hermitian operator obeys the following rule

$$
\langle\psi, \mathcal{O} \varphi\rangle=-\langle\mathcal{O} \psi, \varphi\rangle \Rightarrow \int \psi^{\dagger}(\mathcal{O} \varphi) d x=-\int(\mathcal{O} \psi)^{\dagger} \varphi d x=-\int\left(\psi^{\dagger} \mathcal{O}^{\dagger}\right) \varphi d x(30)
$$

Nevertheless, while in complex and quaternionic quantum mechanics we can define a direct correspondence between Hermitian matrices and Hermitian operators, in octonionic quantum mechanics this is not possible. For example, we shall see that the matricial representation of $e_{m}$ is an anti-Hermitian matrix, but no imaginary unit $e_{m}$ represents an anti-Hermitian operator [22]. In fact, given $\psi: \mathbb{R} \rightarrow \mathbb{O}$ and $\varphi: \mathbb{R} \rightarrow \mathbb{O}$, octonionic functions of a real variable $x$,

$$
\psi(x)=\psi_{0}(x)+\sum_{n=1}^{7} \psi_{n}(x) e_{n} \quad \text { and } \quad \varphi(x)=\varphi_{0}(x)+\sum_{n=1}^{7} \varphi_{n}(x) e_{n},
$$

where each $\psi_{n}$ and each $\varphi_{n}$ are real valued functions, the nonassociativity of the octonionic algebra implies that

$$
\int \psi^{\dagger}\left(e_{m} \varphi\right) d x=\left\langle\psi, e_{m} \varphi\right\rangle \neq-\left\langle e_{m} \psi, \varphi\right\rangle=\int\left(\psi^{\dagger} e_{m}\right) \varphi d x
$$

This contrasts with the situation within complex and quaternionic quantum mechanics. Such a difficulty is overcome by using a complex projection of the scalar product (complex geometry) with respect to one of our imaginary units. We break the symmetry 
between the seven imaginary units $e_{1}, \cdots, e_{7}$ and choose as projection plane the one characterized by $\left(1, e_{1}\right)$. The new scalar product is quickly obtained by performing, in the standard definition, the following substitution:

$$
\int F(x) d x \rightarrow \int_{c} F(x) d x \equiv \frac{1}{2}\left[\int F(x) d x-e_{1}\left(\int F(x) d x\right) e_{1}\right]
$$

Working in Octonionic Quantum Mechanics with complex geometry, $e_{m}$ represents an anti-Hermitian operator. In order to simplify the proof for the imaginary unit $e_{1}$, we write the octonionic functions $\psi$ and $\varphi$ as follows:

$$
\begin{aligned}
& \psi=\tilde{\psi}_{1}+e_{2} \tilde{\psi}_{2}+e_{4} \tilde{\psi}_{3}+e_{6} \tilde{\psi}_{4} \\
& \varphi=\tilde{\varphi}_{1}+e_{2} \tilde{\varphi}_{2}+e_{4} \tilde{\varphi}_{3}+e_{6} \tilde{\varphi}_{4}
\end{aligned}
$$

where $\tilde{\psi}_{m}$ and $\tilde{\varphi}_{m}, m=1,2,3,4$ are complex-valued functions. The anti-hermiticity of $e_{1}$ is recovered if

$$
\int_{c} \psi^{\dagger}\left(e_{1} \varphi\right) d x=-\int_{c}\left(e_{1} \psi\right)^{\dagger} \varphi d x
$$

holds. Algebraic manipulation shows that, after the complex projection, the only non vanishing terms are represented by diagonal terms, that is, terms that contains the functions $\tilde{\psi}_{1}^{\dagger} \tilde{\varphi}_{1}, \tilde{\psi}_{2}^{\dagger} \tilde{\varphi}_{2}, \tilde{\psi}_{3}^{\dagger} \tilde{\varphi}_{3}$ and $\tilde{\psi}_{4}^{\dagger} \tilde{\varphi}_{4}$. In fact, terms like $\tilde{\psi}_{1}^{\dagger} e_{5} \tilde{\varphi}_{3}$ or $\tilde{\psi}_{3}^{\dagger} \tilde{\varphi}_{4}$ are annulled by complex projection,

$$
\begin{aligned}
& \tilde{\psi}_{1}^{\dagger}\left(e_{5} \tilde{\varphi}_{3}\right)=\left(\alpha_{0}-e_{1} \alpha_{1}\right)\left(e_{5} \beta_{0}+e_{4} \beta_{1}\right)=\rho e_{4}+\sigma e_{5} \\
& \left(\tilde{\psi}_{3}^{\dagger} e_{4}\right)\left(-e_{7} \tilde{\varphi}_{4}\right)=-\left(\gamma_{0} e_{4}-\gamma_{1} e_{5}\right)\left(e_{7} \delta_{0}-e_{6} \delta_{1}\right)=\varrho e_{2}+\varsigma e_{3}
\end{aligned}
$$

with $\alpha_{0,1}, \beta_{0,1}, \gamma_{0,1}, \delta_{0,1}, \rho, \sigma, \varrho$ e $\varsigma$ real numbers. The diagonal terms give

$$
\begin{gathered}
\int_{c} \psi^{\dagger}\left(e_{1} \varphi\right) d x=\int_{c} \tilde{\psi}_{1}^{\dagger}\left(e_{1} \tilde{\varphi}_{1}\right)-\left(\tilde{\psi}_{2}^{\dagger} e_{2}\right)\left[e_{1}\left(e_{2} \tilde{\varphi}_{2}\right)\right]-\left(\tilde{\psi}_{3}^{\dagger} e_{4}\right)\left[e_{1}\left(e_{4} \tilde{\varphi}_{3}\right)\right]-\left(\tilde{\psi}_{4}^{\dagger} e_{6}\right)\left[e_{1}\left(e_{6} \tilde{\varphi}_{4}\right)\right] d x \\
\left.\left.\left.-\int_{c}\left(e_{1} \psi\right)^{\dagger} \varphi d x=\int_{c}\left(\tilde{\psi}_{1}^{\dagger} e_{1}\right) \tilde{\varphi}_{1}-\left[\left(\tilde{\psi}_{2}^{\dagger} e_{2}\right) e_{1}\right]\left(e_{2} \tilde{\varphi}_{2}\right)\right]-\left[\left(\tilde{\psi}_{3}^{\dagger} e_{4}\right) e_{1}\right]\left(e_{4} \tilde{\varphi}_{3}\right)\right]-\left[\left(\tilde{\psi}_{4}^{\dagger} e_{6}\right) e_{1}\right]\left(e_{6} \tilde{\varphi}_{4}\right)\right] d x
\end{gathered}
$$

The parentheses above are not relevant since the first term $\tilde{\psi}_{1}^{\dagger} e_{1} \tilde{\varphi}_{1}$ is a complex number and the three remaining terms respectively $\tilde{\psi}_{2}^{\dagger} e_{2} e_{1} e_{2} \tilde{\varphi}_{2}$ (subalgebra 123), $\tilde{\psi}_{3}^{\dagger} e_{4} e_{1} e_{4} \tilde{\varphi}_{3}$ (subalgebra 145) and $\tilde{\psi}_{4}^{\dagger} e_{6} e_{1} e_{6} \tilde{\varphi}_{4}$ (subalgebra 167), are quaternions. This proves that equation (34) holds. Let us discuss the octonionic Hermitian operator. Following the well known definition we have $\mathcal{O}$ is an Hermitian operator if

$$
\langle\psi, \mathcal{O} \varphi\rangle=\langle\mathcal{O} \psi, \varphi\rangle \Rightarrow \int \psi^{\dagger}(\mathcal{O} \varphi) d x=\int(\mathcal{O} \psi)^{\dagger} \varphi d x=\int\left(\psi^{\dagger} \mathcal{O}^{\dagger}\right) \varphi d x(37)
$$

Now, suppose $\mathcal{O}$ an octonionic Hermitian operator and

$$
\mathcal{O} \psi=\psi \lambda, \quad \lambda \in \mathbb{O} .
$$

Applying the definition of hermiticity is easy to prove that $\lambda$ must be real. In fact, let be $\psi=\varphi$, then

$$
\langle\psi, \mathcal{O} \psi\rangle=\langle\mathcal{O} \psi, \psi\rangle \quad \Rightarrow \quad \psi^{\dagger}(\mathcal{O} \psi)=(\mathcal{O} \psi)^{\dagger} \psi=\left(\psi^{\dagger} \mathcal{O}^{\dagger}\right) \psi
$$


The octonionic eigenvalue problem

which gives, after using equation (38),

$$
\psi^{\dagger}(\psi \lambda)=\left(\lambda^{*} \psi^{\dagger}\right) \psi
$$

But octonionic numbers, $o_{1}, o_{2} \in \mathbb{O}$, satisfy the property

$$
o_{1}^{\dagger}\left(o_{1} o_{2}\right)=\left(o_{1}^{\dagger} o_{1}\right) o_{2}=\left(o_{2} o_{1}^{\dagger}\right) o_{1} \text {. }
$$

So, returning to equation (40) and using the property above we have

$$
\psi^{\dagger}(\psi \lambda)=\left(\psi^{\dagger} \psi\right) \lambda=|\psi|^{2} \lambda
$$

and

$$
\left(\lambda^{\dagger} \psi^{\dagger}\right) \psi=\psi^{\dagger}\left(\psi \lambda^{\dagger}\right)=\left(\psi^{\dagger} \psi\right) \lambda^{\dagger}=|\psi|^{2} \lambda^{\dagger}
$$

Since $|\psi|^{2}$ is real we obtain

$$
\lambda=\lambda^{\dagger} \Rightarrow \lambda \in \mathbb{R}
$$

This means that even in the octonionic formalism, given an Hermitian operator its eigenvalues must be real. Now, consider the example given by Dray et al, in [20]. Given $M$ an Hermitian matrix

$$
M=\left(\begin{array}{rc}
1 & e_{4} \\
-e_{4} & 1
\end{array}\right)
$$

we find

$$
\left(\begin{array}{rc}
1 & e_{4} \\
-e_{4} & 1
\end{array}\right)\left(\begin{array}{l}
e_{5} \\
e_{7}
\end{array}\right)=\left(\begin{array}{l}
e_{5} \\
e_{7}
\end{array}\right)\left(1-e_{6}\right)
$$

So, the Hermitian matrix $M$ has an eigenvalue given by $1-e_{6}(\in \mathbb{O})$ and $\left(e_{5} e_{7}\right)^{t}$ is an eigenvector associated with it. Since the matrix is Hermitian we should expect real eigenvalues but this doesn't happen. How can we prove that the matrix $M$ does not represent an Hermitian operator? Consider the example (45) and let be $\psi=\varphi$. So, we have

$$
\begin{gathered}
\psi^{\dagger}(\mathcal{O} \psi)=\psi^{\dagger}(\psi \lambda)=\left(\begin{array}{ll}
-e_{5} & -e_{7}
\end{array}\right)\left[\left(\begin{array}{c}
e_{5} \\
e_{7}
\end{array}\right)\left(1-e_{6}\right)\right]= \\
\left(\begin{array}{ll}
-e_{5} & -e_{7}
\end{array}\right)\left(\begin{array}{c}
e_{5}+e_{3} \\
e_{7}-e_{1}
\end{array}\right)=-e_{5}\left(e_{5}+e_{3}\right)-e_{7}\left(e_{7}-e_{1}\right)=1-e_{6}+1-e_{6}=2-2 e_{6}(46)
\end{gathered}
$$

Now, calculating $(\mathcal{O} \psi)^{\dagger} \psi$ we find

$$
\begin{aligned}
& (\mathcal{O} \psi)^{\dagger} \psi=\left(\begin{array}{c}
e_{5}+e_{3} \\
e_{7}-e_{1}
\end{array}\right)^{\dagger}\left(\begin{array}{l}
e_{5} \\
e_{7}
\end{array}\right)=\left(\begin{array}{ll}
-e_{5}-e_{3} & -e_{7}+e_{1}
\end{array}\right)\left(\begin{array}{l}
e_{5} \\
e_{7}
\end{array}\right)= \\
& -\left(e_{5}+e_{3}\right) e_{5}+\left(e_{1}-e_{7}\right) e_{7}=1+e_{6}+e_{6}+1=2+2 e_{6}
\end{aligned}
$$

Thus, according to definition (37) the operator associated to the Hermitian matrix $M$ is not an Hermitian operator since $\psi^{\dagger}(\mathcal{O} \psi) \neq\left(\psi^{\dagger} \mathcal{O}^{\dagger}\right) \psi$. 
At this point, we can assert that octonionic Hermitian matrices are not associated with Hermitian operators so it's natural to find non real eigenvalues for the matrices. We conclude that octonionic Hermitian operators necessarily have real eigenvalues. Furthermore, a way to reobtain the relation between Hermitian matrices and Hermitian operators is by using the complex projection. It's worthy to observe that the complex projection of the internal product gives the same result. In the example just given

$$
[\langle\psi, \mathcal{O} \psi\rangle]_{\mathbb{C}}=[\langle\mathcal{O} \psi, \psi\rangle]_{\mathbb{C}}=2
$$

\section{Conclusions}

It is well known that amplitudes of probability have to be defined in associative division algebras [5]. Amplitudes of probabilities defined in non-division algebras fail to satisfy the requirement that, in the absence of quantum interference effects, probability amplitude superposition should reduce to probability superposition. The associative law of multiplication is needed to satisfy the completeness formula. Amidst these constraints how can be possible to formulate quantum theories by using wave functions defined in non-division or non-associative algebras? The answer is very simple. The constraints concern the inner product and not the Hilbert space in which we define our wave functions. The amplitudes of probability have to be defined in $\mathbb{C}$ or $\mathbb{H}$ but the vectors in the Hilbert space have no constraint. The choice of quaternionic inner product seems to be best adapted to investigate deviations from the standard complex theory in quantum mechanics and quantum fields [5].

In this paper, we have suggested a solution for the octonionic eigenvalue problem by a formulation based on a coupled equation with two real parameters which play the same role of the complex eigenvalue in complex and quaternionic quantum mechanics. The complex eigenvalue problem in complex and quaternionic quantum mechanic can be obviously solved by a real coupled problem and this should represent the complex and quaternionic limit of the octonionic eigenvalue problem proposed in this article.

An intriguing result obtained from our investigation was that octonionic Hermitian matrices do not necessarily represent octonionic Hermitian operators. This is essentially due to the fact that octonionic Hermitian matrices can have not real eigenvalues. To overcome this problem, we need to introduce a complex geometry, i.e. complex inner products. The natural choice is represented by the use of the imaginary unit $i$ which commutes with the octonionic imaginary units $e_{1,2, \ldots, 7}$.

An interesting application of the material presented in this work can be immediately found in the octonionic formulation of the Dirac equation. The Dirac Hamiltonian [26],

$$
\mathcal{H}=\boldsymbol{\alpha} \cdot \boldsymbol{p} c+\beta m c^{2}, \quad \boldsymbol{p}=-i \hbar \nabla,
$$

which describes the temporal behavior of relativistic particles is given in terms of $4 \times 4$ complex matrices, $\boldsymbol{\alpha}$ and $\beta$, satisfying the Dirac algebra

$$
\{\boldsymbol{\alpha}, \beta\}=0, \quad\left\{\alpha_{m}, \alpha_{n}\right\}=0 \text { for } m \neq n, \quad \alpha_{n}^{2}=\beta^{2}=\mathrm{I}_{4 \times 4} .
$$


By using the quaternionic sub-algebra $e_{1,2,3}$, we can immediately found a complexified quaternionic representation for the $\boldsymbol{\alpha}$ matrices,

$$
\boldsymbol{\alpha}=i \boldsymbol{e}=\left(i e_{1}, i e_{2}, i e_{3}\right) \text {. }
$$

The matrix $\beta$ can be represented by using, for example, the octonionic imaginary unit $e_{4}$,

$$
\beta=i e_{4}
$$

An octonionic representation for the Dirac Hamiltonion is thus given by

$$
\mathcal{H} \rightarrow \hbar c \boldsymbol{e} \cdot \nabla+i m c^{2} e_{4} .
$$

The complexified octonionic solution

$$
\underbrace{\psi_{0}+\boldsymbol{e} \cdot \boldsymbol{\psi}}_{\Psi \in \mathbb{H} \times \mathbb{C}}+e_{4}(\underbrace{\phi_{0}+\boldsymbol{e} \cdot \boldsymbol{\phi}}_{\Phi \in \mathbb{H} \times \mathbb{C}}), \quad \psi_{0}, \boldsymbol{\psi}, \phi_{0}, \boldsymbol{\phi} \in \mathbb{C}(1, i),
$$

by using $\mathbb{C}$ inner products contains in this formulation two orthogonal spinorial solutions, $\Psi$ and $\Phi$, each of one with its 4 complex degrees of freedom represent a Dirac particle. This suggests a natural e simple one dimensional octonionic formulation of the standard model, where two orthogonal spinorial solutions are needed to represent the leptonic and quark doublets [27].

\section{References}

[1] De Leo S and Scolarici G 2000 J. Phys. A: Math. Gen. 33 2971-95.

[2] De Leo S, Scolarici G and Solombrino L 2002 J. Math. Phys. 43 5815-29.

[3] De Leo S and Ducati G 2001 J. Math. Phys. 42 2236-65.

[4] De Leo S and Ducati G 2003 J. Math. Phys. 44 2224-33.

[5] Adler S L 1995 Quaternionic quantum mechanics and quantum fields (Oxford University Press, New York).

[6] Peres A 1979 Phys. Rev. Lett. 42 683-686.

[7] Kaiser H, George E A and Werner S A 1984 Phys. Rev. A 29 2276-79.

[8] Klein A G 1988 Phys. B 151 44-49.

[9] Davies A J and McKellar B H 1989 Phys. Rev. A 40 4209-14.

[10] Davies A J and McKellar B H 1992 Phys. Rev. A 46 3671-75.

[11] De Leo S, Ducati G and Nishi C C 2001 J. Phys. A: Math. Gen. 35 5411-26.

[12] De Leo S and Ducati G 2005 J. Phys. A: Math. Gen. 38 3443-54.

[13] Dixon G M 1994 Division algebras: octonions, quaternions, complex number, and the algebraic design of physics (Kluwer, Dordrecht).

[14] Gürsey F and Tze C H 1996 On the role of division, Jordan and eelated algebras in particle physics (World Scientific, Singapore).

[15] De Leo S and Abdel-Khalek K 1996 Prog. Theor. Phys. 96 823-831.

[16] De Leo S and Abdel-Khalek K 1996 Prog. Theor. Phys. 96 833-845.

[17] Dray T and Manogue C A 1998 Adv. Appl. Cliff. Alg. 8 341-364.

[18] Dray T and Manogue C A 1998 Comput. Phys. Comm. 115 536-547.

[19] Dray T and Manogue C A 1999 Int. J. Theor. Phys. 38 2901-16.

[20] Dray T, Janesky J and Manogue C A 2000 Adv. Appl. Cliff. Alg. 10 193-216.

[21] Dray T, Manogue C A and Okubo S 2002 Alg. Groups Geom. 19, 163-180.

[22] De Leo S and Abdel-Khalek K 1997 J. Math. Phys. 38 582-599. 
[23] Daboul J and Delbourgo R 1999 J. Math. Phys. 40 4134-50.

[24] De Leo S and Ducati G 1999 Int. J. Theor. Phys. 38, 2197-2220.

[25] Rembieliński J 1978 J. Phys. A: Math. Gen. 11 2323-31.

[26] Itzykson C and Zuber J B 1980 Quatum field theory (McGraw-Hill, New York).

[27] Brown L S 1992 Quatum field theory (Cambridge University Press, Bristol). 